\title{
A one-dimensional prescribed curvature equation modeling the corneal shape
}

\author{
Isabel Coelho ${ }^{1,2}$, Chiara Corsato $^{3}$ and Pierpaolo Omari ${ }^{3 *}$
}

\footnotetext{
Correspondence: omari@units.it ${ }^{3}$ Dipartimento di Matematica e Geoscienze, Università degli Studi di Trieste, Via A. Valerio 12/1, Trieste, 34127, Italy

Full list of author information is available at the end of the article
}

\begin{abstract}
We prove existence, uniqueness, and stability of solutions of the prescribed curvature problem $\left(u^{\prime} / \sqrt{\left.1+u^{\prime 2}\right)^{\prime}}=a u-b / \sqrt{1+u^{\prime 2}}\right.$ in $[0,1], u^{\prime}(0)=u(1)=0$, for any given $a>0$ and $b>0$. We also develop a linear monotone iterative scheme for approximating the solution. This equation has been proposed as a model of the corneal shape in the recent paper (Okrasiński and Płociniczak in Nonlinear Anal., Real World Appl.

13:1498-1505, 2012), where a simplified version obtained by partial linearization has been investigated.
\end{abstract}

Keywords: mean curvature equation; mixed boundary condition; positive solution; existence; uniqueness; linear stability; order stability; Lyapunov stability; lower and upper solutions; monotone approximation; topological degree

\section{Introduction}

In this paper we study existence, uniqueness, stability, and approximation of classical solutions of the one-dimensional prescribed curvature problem

$$
\left\{\begin{array}{l}
\left(\frac{u^{\prime}}{\sqrt{1+u^{\prime 2}}}\right)^{\prime}=a u-\frac{b}{\sqrt{1+u^{\prime 2}}} \quad \text { in }[0,1], \\
u^{\prime}(0)=u(1)=0,
\end{array}\right.
$$

where $a>0$ and $b>0$ are given constants. This problem, together with its $N$-dimensional counterpart

$$
\begin{cases}\operatorname{div}\left(\frac{\nabla u}{\sqrt{1+|\nabla u|^{2}}}\right)=a u-\frac{b}{\sqrt{1+|\nabla u|^{2}}} & \text { in } \Omega, \\ u=0 & \text { on } \partial \Omega\end{cases}
$$

has been proposed in [1-4] as a mathematical model for the geometry of the human cornea. However, in these papers a simplified version of (2) has been investigated, where the mean curvature operator $\operatorname{div}\left(\nabla u / \sqrt{1+|\nabla u|^{2}}\right)$ has been replaced by its linearization $\operatorname{div}(\nabla u)$ around 0 . In particular, it has been proved in [1] that, if $b \in] 0, \frac{3 \sqrt{3}}{2} \frac{\sqrt{a}}{\tanh \sqrt{a}}$ [, then the problem

$$
\left\{\begin{array}{l}
u^{\prime \prime}=a u-\frac{b}{\sqrt{1+u^{\prime 2}}} \quad \text { in }[0,1], \\
u^{\prime}(0)=u(1)=0
\end{array}\right.
$$

\section{照 Springer}

O2014 Coelho et al; i licensee Springer. This is an Open Access article distributed under the terms of the Creative Commons Attribution License (http://creativecommons.org/licenses/by/2.0), which permits unrestricted use, distribution, and reproduction in any medium, provided the original work is properly cited. 
has a unique solution which is the limit of a sequence of successive approximations. The above limitations on the parameters have recently been removed in [4].

Unlike all these works we tackle here the fully nonlinear problem (1) and we prove the existence of a unique solution for the whole range of positive parameters $a, b$. The study of problem (1) requires some care because, even if pairs of constant lower and upper solutions can easily be exhibited, the presence of the curvature term rules out in general the possibility of applying the standard existence results, due to the possible occurrence of derivative blow-up phenomena (see, e.g., [5]). On the other hand, the non-variational structure of (1) puts the problem, as it stands, out of the scope of the methods developed in [6-8] for the curvature equation. Nevertheless, we show that an a priori bound in $C^{1}$ for all possible solutions can be obtained by an elementary, but delicate, argument which exploits the qualitative properties - positivity, monotonicity, and concavity - of the solutions themselves. These estimates eventually enable us to use a degree argument in order to prove the existence of solutions. The proof of the uniqueness is then based on suitable fixed point index calculations, which are performed via linearization. A similar approach, applied to an associated evolutionary problem, is exploited for detecting the linear stability of the solution.

Next, taking inspiration from $[9,10]$, we develop a linear iterative scheme for approximating the solution by two monotone sequences of strict lower and upper solutions, starting from an explicit pair of constant lower and upper solutions. These two sequences, besides providing accurate two-sided bounds on the solution, yield the strict order stability and hence, according to [11], the (Lyapunov) asymptotic stability of the solution itself, yielding as well an explicitly computable estimate of its basin of attractivity. We finally illustrate the use of this approximation scheme in order to compute numerically the solution $u$ of (1) for the same choice of the parameters $a$ and $b$ as the one considered in [1].

We finally mention that part of our results extends to the general $N$-dimensional problem (2); this topic will be discussed elsewhere.

\section{Existence, qualitative properties and approximation}

In this section we are concerned with the study of the existence, the qualitative properties and the approximation of classical solutions, i.e., belonging to $C^{2}([0,1])$, of problem (1), where $a>0$ and $b>0$ are fixed constants. Clearly, problem (1) can be written in the equivalent form

$$
\left\{\begin{array}{l}
u^{\prime \prime}=a u\left(1+u^{\prime 2}\right)^{3 / 2}-b\left(1+u^{\prime 2}\right) \quad \text { in }[0,1], \\
u^{\prime}(0)=u(1)=0 .
\end{array}\right.
$$

Let us set for convenience, for all $(s, \xi) \in \mathbb{R}^{2}$,

$$
f(s, \xi)=a s\left(1+\xi^{2}\right)^{3 / 2}-b\left(1+\xi^{2}\right) .
$$

It is obvious that, due to the symmetry properties of the function $f$, the mixed problem (4) is equivalent to the Dirichlet problem

$$
\left\{\begin{array}{l}
u^{\prime \prime}=f\left(u, u^{\prime}\right) \quad \text { in }[-1,1] \\
u(-1)=u(1)=0
\end{array}\right.
$$


Notations As usual, for functions $u, v \in C^{0}([c, d])$, we write $u \leq v$ in $[c, d]$ if $u(t) \leq v(t)$ for all $t \in[c, d]$ and $u<v$ in $[c, d]$ if $u \leq v$ and $u \neq v$. We also write $u \ll v$ in $[c, d]$ if $u(t)<v(t)$ for all $t \in\left[c, d\left[\right.\right.$ and, if $u(d)=v(d), D_{-} u(d)>D^{-} v(d)$, where $D_{-}, D^{-}$denote the left Dini derivatives; this is equivalent to requiring that there exists $\delta>0$ such that $v(t)-u(t) \geq$ $\delta(d-t)$ for all $t \in[c, d]$. Whenever no confusion occurs, we omit the indication of the interval.

\section{Existence, uniqueness, and linear stability}

We start with a preliminary result, where some properties of the possible solutions of problem (1) are highlighted.

Lemma 2.1 The following assertions hold.

(i) Any solution $u$ of (1) satisfies $u(t)>0$ for all $t \in\left[0,1\left[\right.\right.$ and $u(t)<\frac{b}{a}$ for all $t \in[0,1]$.

(ii) Any solution $u$ of (1) is such that $u^{\prime}(t)<0$ and $u^{\prime \prime}(t) \leq 0$ for all $\left.\left.t \in\right] 0,1\right]$.

(iii) Any solution $u$ of $(1)$ is such that $u^{\prime}(t)>-R$ for all $t \in[0,1]$, where $R=\sqrt{\exp \left(\frac{2 b^{2}}{a}\right)-1}$.

Proof In the following steps $u$ denotes a solution of (1), or equivalently of (4). From the equation in (4) it follows that, if $\hat{t} \in[0,1]$ is such that $u^{\prime}(\hat{t})=0$ and $u(\hat{t}) \neq \frac{b}{a}$, then

$$
u^{\prime \prime}(\hat{t})\left(u(\hat{t})-\frac{b}{a}\right)>0
$$

Step 1. Proof of (i). Let us first show that $u \geq 0$ in $[0,1]$. Assume by contradiction that $\min _{[0,1]} u=u(\hat{t})<0$. The boundary condition $u(1)=0$ implies that $\hat{t} \in[0,1[$. Suppose that $\hat{t}=0$. We have $u(0)<0$ and $u^{\prime}(0)=0$. Condition (6) yields $u^{\prime \prime}(0)<0$. Hence there exists $\delta>0$ such that $u^{\prime}(t)<0$ for all $\left.t \in\right] 0, \delta$ [ and therefore $u(t)<u(0)=\min _{[0,1]} u$ for all $\left.t \in\right] 0, \delta[$, which is a contradiction. Now suppose that $\hat{t} \in] 0,1\left[\right.$. We have $u(\hat{t})<0$ and $u^{\prime}(\hat{t})=0$ and condition (6) yields again a contradiction. Hence we conclude that $u \geq 0$ in $[0,1]$. In a completely similar way we prove that $u \leq \frac{b}{a}$ in $[0,1]$.

Next, in order to prove that

$$
u(t)>0
$$

for all $t \in\left[0,1\left[\right.\right.$, it is sufficient to note that, if $u(\hat{t})=u^{\prime}(\hat{t})=0$ for some $\hat{t} \in[0,1[$, then (6) would yield $u^{\prime \prime}(\hat{t})<0$, which is impossible. Moreover, as the constant function $\frac{b}{a}$ is a solution of the equation in (4), the uniqueness of solutions for any Cauchy problem associated with this equation implies that

$$
u(t)<\frac{b}{a},
$$

for all $t \in[0,1]$.

Step 2. Proof of (ii). As $u^{\prime}(0)=0$, assertion (i) implies that there exists $\delta>0$ such that $u^{\prime \prime}(t)<0$ for all $t \in\left[0, \delta\left[\right.\right.$ and $u^{\prime}(t)<0$ for all $\left.t \in\right] 0, \delta[$. Let us show that

$$
u^{\prime}(t)<0,
$$


for all $t \in] 0,1]$. If this is not the case, set $\left.\hat{t}=\min \{t \in] 0,1]: u^{\prime}(t)=0\right\}$. Then, by (6), we have $u^{\prime \prime}(\hat{t})<0$ and hence there exists $\eta>0$ such that $u^{\prime}(t)>u^{\prime}(\hat{t})=0$, for all $\left.t \in\right] \hat{t}-\eta, \hat{t}[$, which contradicts the definition of $\hat{t}$.

Let us now prove that

$$
u^{\prime \prime} \leq 0
$$

in $[0,1]$. By contradiction, assume that there exists $\bar{t} \in] 0,1]$ such that $u^{\prime \prime}(\bar{t})>0$. As $u^{\prime \prime}(0)<0$, there exist $\left.t_{0} \in\right] 0, \bar{t}\left[\right.$ and $\delta>0$ such that $u^{\prime \prime}\left(t_{0}\right)=0$ and $u^{\prime \prime}(t)>0$ for all $t \in$ ]$t_{0}, t_{0}+\delta\left[\right.$. Since we have $u^{\prime}(t) u^{\prime \prime}(t)<0$ for all $\left.t \in\right] t_{0}, t_{0}+\delta\left[\right.$, the function $-b\left(1+u^{\prime 2}\right)^{-\frac{1}{2}}$ is decreasing in $] t_{0}, t_{0}+\delta[$. We also know that the function $a u$ is decreasing in $[0,1]$. Hence the function $a u-b\left(1+u^{\prime 2}\right)^{-\frac{1}{2}}$ is decreasing in $] t_{0}, t_{0}+\delta\left[\right.$. On the other hand, as $u^{\prime \prime}\left(t_{0}\right)=0$, from the equation in (4) we get

$$
a u\left(t_{0}\right)-b\left(1+\left(u^{\prime}\left(t_{0}\right)\right)^{2}\right)^{-\frac{1}{2}}=0,
$$

and therefore

$$
a u(t)-b\left(1+\left(u^{\prime}(t)\right)^{2}\right)^{-\frac{1}{2}}<0
$$

for all $t \in] t_{0}, t_{0}+\delta\left[\right.$. Then the equation in (4) yields $u^{\prime \prime}(t)<0$ for all $\left.t \in\right] t_{0}, t_{0}+\delta[$, which is a contradiction.

Step 3. Proof of (iii). Since by assertion (i) $u \geq 0$ in $[0,1]$, we get $f\left(u, u^{\prime}\right) \geq-b\left(1+u^{\prime 2}\right)$ in $[0,1]$, and then, from the equation in (4), we conclude that

$$
\frac{u^{\prime \prime}}{1+u^{\prime 2}} \geq-b
$$

in $[0,1]$. Multiplying by $u^{\prime}$, where $u^{\prime} \leq 0$ in $[0,1]$ by assertion (ii), and integrating between 0 and 1 , we obtain

$$
\int_{0}^{1} \frac{u^{\prime} u^{\prime \prime}}{1+u^{\prime 2}} d t \leq \int_{0}^{1}-b u^{\prime} d t
$$

On the one hand, using the boundary condition $u^{\prime}(0)=0$ we have

$$
\int_{0}^{1} \frac{u^{\prime} u^{\prime \prime}}{1+u^{\prime 2}} d t=\frac{1}{2} \ln \left(1+\left(u^{\prime}(1)\right)^{2}\right)
$$

On the other hand, the boundary condition $u(1)=0$ and assertion (i) imply

$$
\int_{0}^{1}-b u^{\prime} d t<\frac{b^{2}}{a}
$$

In conclusion, setting

$$
R=\sqrt{\exp \left(\frac{2 b^{2}}{a}\right)-1},
$$


we get $u^{\prime}(1)>-R$. Since $u^{\prime}$ is non-increasing, we conclude

$$
u^{\prime}(t)>-R
$$

for all $t \in[0,1]$.

We are now in position to prove the existence of a unique solution of problem (1), which is linearly stable.

Theorem 2.2 Let $a>0$ and $b>0$ be given. Then there exists a unique solution $u$ of (1) and it satisfies the conditions (i), (ii), and (iii). Further, $u$ is linearly stable as an equilibrium of the parabolic problem

$$
\begin{cases}\partial_{\tau} u-\partial_{t t} u+f\left(u, \partial_{t} u\right)=0 & \text { in }] 0,1[\times] 0,+\infty[ \\ \partial_{t} u(0, \tau)=u(1, \tau)=0 & \text { in }] 0,+\infty[\end{cases}
$$

Proof The proof is divided into three steps.

Step 1. Existence. Let us prove that there exists at least one solution of (1), or equivalenty of (4). Let $\mathfrak{K}: C^{0}([0,1]) \rightarrow C^{1}([0,1])$ be the operator which associates with any $h \in C^{0}([0,1])$ the unique solution $w$ of

$$
\left\{\begin{array}{l}
w^{\prime \prime}=h \quad \text { in }[0,1] \\
w^{\prime}(0)=w(1)=0 .
\end{array}\right.
$$

Clearly, $\mathfrak{K}$ is completely continuous. Moreover, let $\mathfrak{F}: C^{1}([0,1]) \rightarrow C^{0}([0,1])$ be the Nemitski operator associated with $f$, i.e., $\mathfrak{F}(w)=f\left(w, w^{\prime}\right)$ for any $w \in C^{1}([0,1])$. The operator $\mathfrak{F}$ is continuous and maps bounded sets into bounded sets. Introduce the open bounded subset of $C^{1}([0,1])$

$$
\mathcal{Q}=\left\{u \in C^{1}([0,1]):\|u\|_{\infty}<\frac{b}{a},\left\|u^{\prime}\right\|_{\infty}<R\right\}
$$

Finally, define a completely continuous operator $\mathfrak{T}: \overline{\mathcal{Q}} \rightarrow C^{1}([0,1])$ by $\mathfrak{T}=\mathfrak{K} \circ \mathfrak{F}$. The fixed points of $\mathfrak{T}$ are precisely the solutions of (4).

An inspection of the assertions of Lemma 2.1 shows that, if $u \in \overline{\mathcal{Q}}$ satisfies, for some $\lambda \in[0,1]$,

$$
u=\lambda \mathfrak{T}(u),
$$

i.e.,

$$
\left\{\begin{array}{l}
u^{\prime \prime}=\lambda a u\left(1+u^{\prime 2}\right)^{3 / 2}-\lambda b\left(1+u^{\prime 2}\right) \quad \text { in }[0,1] \\
u^{\prime}(0)=u(1)=0
\end{array}\right.
$$

then $u \in \mathcal{Q}$. The invariance property of the degree under homotopy implies that

$$
\operatorname{deg}(\mathfrak{I}-\mathfrak{T}, \mathcal{Q}, 0)=\operatorname{deg}(\mathfrak{I}, \mathcal{Q}, 0)=1,
$$


where $\mathfrak{I}$ stands for the identity operator. Therefore there exists a fixed point $u \in \mathcal{Q}$ of $\mathfrak{T}$, which is a solution of (4).

By Lemma 2.1, $u$ satisfies the conditions (i), (ii), and (iii).

Step 2. Uniqueness. Set $\Phi=\mathfrak{I}-\mathfrak{T}$. As the function $f: \mathbb{R}^{2} \rightarrow \mathbb{R}$ is of class $C^{1}$, the operators $\mathfrak{F}: C^{1}([0,1]) \rightarrow C^{0}([0,1])$ and, hence, $\Phi: C^{1}([0,1]) \rightarrow C^{1}([0,1])$ are of class $C^{1}$, with Fréchet differentials

$$
\mathfrak{F}^{\prime}(u) w=\frac{\partial f}{\partial s}\left(u, u^{\prime}\right) w+\frac{\partial f}{\partial \xi}\left(u, u^{\prime}\right) w^{\prime} \quad \text { and } \quad \Phi^{\prime}(u) w=\left(\mathfrak{I}-\mathfrak{K} \circ \mathfrak{F}^{\prime}(u)\right) w
$$

for any given $u \in C^{1}([0,1])$ and all $w \in C^{1}([0,1])$.

Observe that, for any $u \in C^{1}([0,1]), \Phi^{\prime}(u)$ is invertible. Indeed, let us fix $u \in C^{1}([0,1])$ and assume that $\Phi^{\prime}(u) w=0$ for some $w \in C^{1}([0,1])$. This means that $w$ is the solution of

$$
\left\{\begin{array}{l}
w^{\prime \prime}=\frac{\partial f}{\partial \xi}\left(u, u^{\prime}\right) w^{\prime}+\frac{\partial f}{\partial s}\left(u, u^{\prime}\right) w \quad \text { in }[0,1] \\
w^{\prime}(0)=w(1)=0
\end{array}\right.
$$

Since

$$
\frac{\partial f}{\partial s}(s, \xi)=a\left(1+\xi^{2}\right)^{3 / 2}>0
$$

for all $(s, \xi) \in \mathbb{R}^{2}$, the maximum principle [5, Appendix, Theorem 5.2] implies that $w=0$. Hence the local inversion theorem applies to $\Phi$ at every point $u \in C^{1}([0,1])$ and thus any fixed point of $\mathfrak{T}$ is isolated. The compactness in $C^{1}([0,1])$ of the set $\mathcal{S}$ of all fixed points of $\mathfrak{T}$ then implies that $\mathcal{S}$ is finite, i.e., $\mathcal{S}=\left\{u_{1}, \ldots, u_{N}\right\}$ for some positive integer $N$.

Denote by $B(u, r)$ the open ball in $C^{1}([0,1])$ centered at $u$ and having radius $r$. Pick $r>0$ so small that $B\left(u_{k}, r\right) \subseteq \mathcal{Q}$ for all $k \in\{1, \ldots, N\}$, and $B\left(u_{i}, r\right) \cap B\left(u_{j}, r\right)=\emptyset$ for all $i, j \in\{1, \ldots, N\}$, with $i \neq j$. The excision and the additivity properties of the degree yield

$$
\operatorname{deg}(\Phi, \mathcal{Q}, 0)=\sum_{k=1}^{N} \operatorname{deg}\left(\Phi, B\left(u_{k}, r\right), 0\right)=\sum_{k=1}^{N} i\left(\Phi, u_{k}\right)
$$

where, for each $k \in\{1, \ldots, N\}$,

$$
i\left(\Phi, u_{k}\right)=\operatorname{deg}\left(\Phi, B\left(u_{k}, r\right), 0\right)
$$

denotes the fixed point index of $u_{k}$. Using again (15), we see as above that, for any given $u \in C^{1}([0,1])$ and all $\mu>0$, the problem

$$
\left\{\begin{array}{l}
\mu w^{\prime \prime}=\frac{\partial f}{\partial \xi}\left(u, u^{\prime}\right) w^{\prime}+\frac{\partial f}{\partial s}\left(u, u^{\prime}\right) w \quad \text { in }[0,1] \\
w^{\prime}(0)=w(1)=0
\end{array}\right.
$$

has no non-trivial solution $w$. Accordingly, for any given $u \in C^{1}([0,1])$, the operator $\mathfrak{T}^{\prime}(u)$ does not have any eigenvalue $\mu>0$. Therefore, we infer from [12, Theorem 3.20] that

$$
i\left(\Phi, u_{k}\right)=1
$$


for all $k \in\{1, \ldots, N\}$. Finally, by (13), (16), and (17) we conclude that $N=1$, i.e., there is a unique solution $u$ of problem (4).

Step 3. Linear stability. The solution $u$ of (4) is an equilibrium of the parabolic problem (11), in particular, it is a 1-periodic solution of (11). In order to show that $u$ is linearly stable, and hence locally exponentially asymptotically stable, it is enough, after a standard cut-off argument, to show that the eigenvalue problem

$$
\begin{cases}\partial_{\tau} w-\partial_{t t} w+\frac{\partial f}{\partial \xi}\left(u, \partial_{t} u\right) \partial_{t} w+\frac{\partial f}{\partial s}\left(u, \partial_{t} u\right) w=\mu w & \text { in }] 0,1[\times \mathbb{R} \\ \partial_{t} w(0, \tau)=w(1, \tau)=0 & \text { in } \mathbb{R}, \\ w(t, \tau)=w(t, \tau+1) & \text { in }] 0,1[\times \mathbb{R}\end{cases}
$$

does not have any eigenvalue $\mu \leq 0$ (see, e.g., [13, Chapter III.23]), or [14, Chapter V.22]). Indeed, if $w$ is a solution of (18) for some $\mu \leq 0$, then using again condition (15), together with the interior form of the parabolic maximum principle and the Hopf boundary point lemma (see, e.g., [14, Chapter III.13]), we conclude that $w=0$.

\section{Monotone approximation and order stability}

In this section we discuss approximation and stability of the solution of (1), or equivalently of (4). To this end, we define a linear iterative scheme that allows one to construct an increasing sequence of strict lower solutions and a decreasing sequence of strict upper solutions of (4) which converge in $C^{2}([0,1])$ to the unique solution $u$ of (4), that is, of (1). Then, according to $[11,13]$, we see that $u$ is strictly order stable from above and from below and hence it is (Lyapunov) asymptotically stable as an equilibrium of the parabolic problem (11). In addition, the converging sequences of lower and upper solutions provide explicitly computable estimates of the basin of attractivity of the solution.

Lower and upper solutions Let us consider the problem

$$
\left\{\begin{array}{l}
u^{\prime \prime}=g\left(t, u, u^{\prime}\right) \quad \text { in }[0,1] \\
u^{\prime}(0)=u(1)=0
\end{array}\right.
$$

where $g:[0,1] \times \mathbb{R} \times \mathbb{R} \rightarrow \mathbb{R}$ is locally Lipschitz continuous. A lower solution of (19) is a function $\alpha \in C^{2}([0,1])$ which satisfies

$$
\left\{\begin{array}{l}
\alpha^{\prime \prime} \geq g\left(t, \alpha, \alpha^{\prime}\right) \quad \text { in }[0,1] \\
\alpha^{\prime}(0) \geq 0 \geq \alpha(1)
\end{array}\right.
$$

Similarly an upper solution of (19) is a function $\beta \in C^{2}([0,1])$ which satisfies

$$
\left\{\begin{array}{l}
\beta^{\prime \prime} \leq g\left(t, \beta, \beta^{\prime}\right) \quad \text { in }[0,1] \\
\beta^{\prime}(0) \leq 0 \leq \beta(1)
\end{array}\right.
$$

Remark 2.1 The Lipschitz character of $g$ implies (see [5, Chapter 3, Proposition 1.7, Proposition 2.7]) that a lower solution $\alpha$ of (19), which is not a solution, is a strict lower solution, that is, any solution $u$ of (19), such that $u \geq \alpha$, satisfies $u \gg \alpha$ in $[0,1]$. Similarly, 
an upper solution $\beta$ of (19), which is not a solution, is a strict upper solution, that is, any solution $u$ of (19), such that $u \leq \beta$, satisfies $u \ll \beta$ in $[0,1]$.

Remark 2.2 Any constant $\alpha \leq 0$ is a strict lower solution of (4) and any constant $\beta \geq \frac{b}{a}$ is a strict upper solution of (4). In particular, one can choose $\alpha=0$ and $\beta=\frac{b}{a}$. We wish to point out that, with this choice of lower and upper solutions, the existence of at least one solution $u$ of problem (4) between $\alpha$ and $\beta$ can be alternatively achieved by applying [5, Chapter 2, Theorem 3.1]; the relevant observations being here the facts that $u^{\prime}(0)=0$, $u^{\prime}(1) \leq 0$ and $f$ satisfies the one-sided Nagumo condition

$$
f(s, \xi) \geq-b\left(1+\xi^{2}\right)
$$

for all $(s, \xi) \in \mathbb{R}^{2}$ such that $0 \leq s \leq \frac{b}{a}$. We point out that one-sided Nagumo conditions were introduced for the first time by Kiguradze in [15].

Let us consider the following modified problem:

$$
\left\{\begin{array}{l}
u^{\prime \prime}=\hat{f}\left(u, u^{\prime}\right) \quad \text { in }[0,1] \\
u^{\prime}(0)=u(1)=0 .
\end{array}\right.
$$

Here $\hat{f}: \mathbb{R}^{2} \rightarrow \mathbb{R}$ is defined as follows. We first introduce an auxiliary function $\tilde{f}$ by setting, for all $(s, \xi) \in \mathbb{R}^{2}$,

$$
\tilde{f}(s, \xi)= \begin{cases}f(s, \xi) & \text { if }|\xi| \leq R \\ \left(\text { as }-\frac{b}{\sqrt{1+\xi^{2}}}\right)\left(1+R^{2}\right)^{3 / 2} & \text { if }|\xi|>R\end{cases}
$$

where $R$ is defined in (10). Then we set, for all $(s, \xi) \in \mathbb{R}^{2}$,

$$
\hat{f}(s, \xi)= \begin{cases}\tilde{f}(0, \xi) & \text { if } s<0 \\ \tilde{f}(s, \xi) & \text { if } 0 \leq s<\frac{b}{a} \\ \tilde{f}\left(\frac{b}{a}, \xi\right) & \text { if } \frac{b}{a} \leq s\end{cases}
$$

The function $\hat{f}$ is locally Lipschitz continuous and satisfies the following conditions:

$\left(\mathrm{h}_{1}\right)$ there exists $\hat{a}>0$ such that

$$
0 \leq \hat{f}\left(s_{2}, \xi\right)-\hat{f}\left(s_{1}, \xi\right) \leq \hat{a}\left(s_{2}-s_{1}\right)
$$

$$
\text { holds for all }\left(s_{1}, \xi\right),\left(s_{2}, \xi\right) \in \mathbb{R}^{2} \text {, with } s_{1} \leq s_{2} ;
$$

$\left(\mathrm{h}_{2}\right)$ there exists $N>0$ such that

$$
\left|\hat{f}\left(s, \xi_{2}\right)-\hat{f}\left(s, \xi_{1}\right)\right| \leq N\left|\xi_{2}-\xi_{1}\right|
$$

holds for all $\left(s, \xi_{1}\right),\left(s, \xi_{2}\right) \in \mathbb{R}^{2}$.

We can choose $\hat{a}=a\left(1+R^{2}\right)^{3 / 2}$ in $\left(\mathrm{h}_{1}\right)$ and $N=\max \left\{b \sqrt{1+R^{2}}, b R\left(2+3 \sqrt{1+R^{2}}\right)\right\}$ in $\left(\mathrm{h}_{2}\right)$. 
Remark 2.3 Any constant $\alpha \leq 0$ and any constant $\beta \geq \frac{b}{a}$ are, respectively, a strict lower and a strict upper solution of (21) too.

Lemma 2.3 A function $u \in C^{2}([0,1])$ is a solution of (21) if and only if it is a solution of (1).

Proof Let $u$ be a solution of (21). In order to prove that $u$ is also a solution of (1), or equivalently of (4), it is sufficient to show that $u$ satisfies $0 \leq u \leq \frac{b}{a}$ and $-R \leq u^{\prime} \leq 0$ in $[0,1]$.

The function $\hat{f}$ satisfies the following conditions:

$$
\hat{f}(s, \xi)<0, \quad \text { for all } s \leq 0 \text { and all } \xi \in \mathbb{R},
$$

and

$$
\hat{f}(s, \xi) \geq 0, \quad \text { for all } s \geq \frac{b}{a} \text { and all } \xi \in \mathbb{R}
$$

It can be verified, proceeding as in the proof of Lemma 2.1 and using the maximum principle, that $0 \leq u \leq \frac{b}{a}$ and hence $0 \ll u \ll \frac{b}{a}$ in $[0,1]$.

Next we prove that $-R \leq u^{\prime} \leq 0$ in $[0,1]$. The proof of assertion (ii) in Lemma 2.1 can be repeated verbatim in order to show that $u^{\prime}(t)<0$ for all $\left.\left.t \in\right] 0,1\right]$ and $u^{\prime \prime} \leq 0$ in $[0,1]$. Assume now that there exists $\left.\left.t_{0} \in\right] 0,1\right]$ such that $u^{\prime}\left(t_{0}\right)=-R$. In particular, we have $-R \leq$ $u^{\prime} \leq 0$ in $\left[0, t_{0}\right]$ and $u^{\prime} \leq-R$ in $\left[t_{0}, 1\right]$. By definition of $\hat{f}, u$ satisfies

$$
u^{\prime \prime}=\left(a u-\frac{b}{\sqrt{1+u^{\prime 2}}}\right)\left(1+u^{\prime 2}\right)^{3 / 2}
$$

in $\left[0, t_{0}\right]$ and

$$
u^{\prime \prime}=\left(a u-\frac{b}{\sqrt{1+u^{\prime 2}}}\right)\left(1+R^{2}\right)^{3 / 2}
$$

in $\left[t_{0}, 1\right]$. As $u \geq 0$ and $u^{\prime} \leq 0$ in $[0,1]$, we easily get from (23)

$$
\int_{0}^{t_{0}} \frac{u^{\prime} u^{\prime \prime}}{1+u^{\prime 2}} d t \leq \int_{0}^{t_{0}}-b u^{\prime} d t
$$

From (24), using again $u \geq 0$ and $u^{\prime} \leq 0$ in $[0,1]$, we obtain

$$
u^{\prime \prime} \geq-b \frac{\left(1+R^{2}\right)^{3 / 2}}{\sqrt{1+u^{\prime 2}}}
$$

and hence

$$
u^{\prime} u^{\prime \prime} \frac{\sqrt{1+u^{\prime 2}}}{\left(1+R^{2}\right)^{3 / 2}} \leq-b u^{\prime}
$$

in $\left[t_{0}, 1\right]$. Since

$$
\frac{1}{1+u^{\prime 2}}=\frac{\sqrt{1+u^{\prime 2}}}{\left(1+u^{\prime 2}\right)^{3 / 2}} \leq \frac{\sqrt{1+u^{\prime 2}}}{\left(1+R^{2}\right)^{3 / 2}}
$$


in $\left[t_{0}, 1\right]$ and $u^{\prime} u^{\prime \prime} \geq 0$ in $[0,1]$, we finally get from $(26)$

$$
\int_{t_{0}}^{1} \frac{u^{\prime} u^{\prime \prime}}{1+u^{\prime 2}} d t \leq \int_{t_{0}}^{1}-b u^{\prime} d t
$$

Combining (25) and (27) yields

$$
\int_{0}^{1} \frac{u^{\prime} u^{\prime \prime}}{1+u^{\prime 2}} d t \leq \int_{0}^{1}-b u^{\prime} d t
$$

which is precisely (9) in Step 3 of the proof of Lemma 2.1. As there we conclude that $u^{\prime} \geq-R$ in $[0,1]$. Accordingly, $u$ is a solution of (4).

Conversely, the definition of $\hat{f}$ implies that any solution of (1), or equivalently of (4), is a solution of (21) as well.

Let us consider the following auxiliary linear problem:

$$
\left\{\begin{array}{l}
w^{\prime \prime}+\sqrt{L} w^{\prime}-L w=h \quad \text { in }[0,1] \\
w^{\prime}(0)=0 \\
w(1)=m .
\end{array}\right.
$$

Here, $h:[0,1] \rightarrow \mathbb{R}$ is a continuous function and $L>0$ and $m \in \mathbb{R}$ are given constants. Notice that problem $(28)$ has a unique solution $w \in C^{2}([0,1])$. The following result is inspired from [9] and [10, Chapter 5].

Lemma 2.4 There exists $L_{0}>0$ such that for all $L \geq L_{0}$, for all $h \in C^{0}([0,1])$, with $h \leq 0$ in $[0,1]$, and for all $m \geq 0$, the solution $w$ of (28) satisfies

$$
(\hat{a}-L) w+\left(N \operatorname{sgn}\left(w^{\prime}\right)+\sqrt{L}\right) w^{\prime} \leq 0 \quad \text { in }[0,1] .
$$

In addition, if $h<0$ in $[0,1]$ or $m>0$, then

$$
(\hat{a}-L) w(t)+\left(N \operatorname{sgn}\left(w^{\prime}(t)\right)+\sqrt{L}\right) w^{\prime}(t)<0 \quad \text { for all } t \in[0,1] .
$$

Proof Let us denote by $w_{1}$ and $w_{2}$ the respective solutions of

$$
\left\{\begin{array}{l}
w_{1}^{\prime \prime}+\sqrt{L} w_{1}^{\prime}-L w_{1}=0 \quad \text { in }[0,1] \\
w_{1}(0)=1 \\
w_{1}^{\prime}(0)=0
\end{array}\right.
$$

and

$$
\left\{\begin{array}{l}
w_{2}^{\prime \prime}+\sqrt{L} w_{2}^{\prime}-L w_{2}=0 \quad \text { in }[0,1] \\
w_{2}(1)=0 \\
w_{2}^{\prime}(1)=-1 .
\end{array}\right.
$$


Step 1 . The functions $w_{1}$ and $w_{2}$ satisfy

$$
\begin{array}{ll}
w_{1}^{\prime}(t)>0 & \text { for all } t \in] 0,1], \\
w_{1}(t)>0 & \text { for all } t \in[0,1]
\end{array}
$$

and

$$
\begin{array}{ll}
w_{2}^{\prime}(t)<0 & \text { for all } t \in[0,1], \\
w_{2}(t)>0 & \text { for all } t \in[0,1[.
\end{array}
$$

A simple computation yields

$$
\begin{aligned}
& w_{1}(t)=A \exp \left(c_{1} t \sqrt{L}\right)+B \exp \left(c_{2} t \sqrt{L}\right), \\
& w_{2}(t)=C \exp \left(c_{1} t \sqrt{L}\right)+D \exp \left(c_{2} t \sqrt{L}\right),
\end{aligned}
$$

where

$$
\begin{aligned}
& c_{1}=\frac{\sqrt{5}-1}{2}, \quad c_{2}=-\frac{\sqrt{5}+1}{2}, \\
& A=\frac{\sqrt{5}+1}{2 \sqrt{5}}, \quad B=\frac{\sqrt{5}-1}{2 \sqrt{5}}, \\
& C=-\frac{\exp \left(\frac{1-\sqrt{5}}{2} \sqrt{L}\right)}{\sqrt{5 L}}, \quad D=\frac{\exp \left(\frac{\sqrt{5}+1}{2} \sqrt{L}\right)}{\sqrt{5 L}} .
\end{aligned}
$$

The conclusion then easily follows by direct calculations.

Step 2. There exists $L_{0}>0$ such that, for any $L \geq L_{0}$, the following inequalities hold:

$$
W_{1}(t)=(\hat{a}-L) w_{1}(t)+(N+\sqrt{L}) w_{1}^{\prime}(t)<0 \quad \text { for all } t \in[0,1]
$$

and

$$
W_{2}(t)=(\hat{a}-L) w_{2}(t)+(-N+\sqrt{L}) w_{2}^{\prime}(t)<0 \quad \text { for all } t \in[0,1] .
$$

Let us first show that (37) holds. We have, for all $t \in[0,1]$,

$$
\begin{aligned}
W_{1}(t)= & (\hat{a}-L) w_{1}(t)+(N+\sqrt{L}) w_{1}^{\prime}(t) \\
= & \left((\hat{a}-L)+c_{1}(N+\sqrt{L}) \sqrt{L}\right) A \exp \left(c_{1} t \sqrt{L}\right) \\
& +\left((\hat{a}-L)+c_{2}(N+\sqrt{L}) \sqrt{L}\right) B \exp \left(c_{2} t \sqrt{L}\right) \\
= & \left(-L\left(1-c_{1}\right)+c_{1} N \sqrt{L}+\hat{a}\right) A \exp \left(c_{1} t \sqrt{L}\right) \\
& +\left(-L\left(1-c_{2}\right)+c_{2} N \sqrt{L}+\hat{a}\right) B \exp \left(c_{2} t \sqrt{L}\right) .
\end{aligned}
$$

Since $c_{1}<1$ and $c_{2}<0$, we can conclude that, for any $L>0$ sufficiently large, $W_{1}(t)<0$ for all $t \in[0,1]$. Namely, if we set

$$
L_{0}=\frac{\left(c_{1} N\right)^{2}+2 \hat{a}\left(1-c_{1}\right)+c_{1} N \sqrt{\left(c_{1} N\right)^{2}+4 \hat{a}\left(1-c_{1}\right)}}{2\left(1-c_{1}\right)^{2}}
$$


and we take $L \geq L_{0}$, we have

$$
-L\left(1-c_{1}\right)+c_{1} N \sqrt{L}+\hat{a} \leq 0 .
$$

Moreover, since $L_{0}>\hat{a}$, the inequality

$$
-L\left(1-c_{2}\right)+c_{2} N \sqrt{L}+\hat{a}<0
$$

holds as well. This yields the validity of (37).

As for (38), by the sign properties of $w_{2}$ and $w_{2}^{\prime}$, we see that $W_{2}(t)<0$ for all $t \in[0,1]$ provided that $L>\max \left\{\hat{a}, N^{2}\right\}$ : this condition holds as $L_{0}>\max \left\{\hat{a}, N^{2}\right\}$.

Fix now $L \geq L_{0}, h \in C^{0}([0,1])$, with $h \leq 0$ in $[0,1]$, and $m \geq 0$. Let $w$ be the solution of problem (28). If $h=0$ in $[0,1]$ and $m=0$, then (29) trivially follows. Therefore suppose that $h<0$ in $[0,1]$ or $m>0$. We can express $w$ as

$$
w(t)=w_{2}(t) \int_{0}^{t} \frac{w_{1} h}{w_{2}^{\prime} w_{1}-w_{2} w_{1}^{\prime}} d s+w_{1}(t)\left[\int_{t}^{1} \frac{w_{2} h}{w_{2}^{\prime} w_{1}-w_{2} w_{1}^{\prime}} d s+\frac{m}{w_{1}(1)}\right],
$$

for all $t \in[0,1]$. Inequality (29) now reads

$$
\begin{aligned}
(\hat{a}-L) w(t)+\left(N \operatorname{sgn}\left(w^{\prime}(t)\right)+\sqrt{L}\right) w^{\prime}(t) \\
=\left((\hat{a}-L) w_{2}(t)+\left(N \operatorname{sgn}\left(w^{\prime}(t)\right)+\sqrt{L}\right) w_{2}^{\prime}(t)\right) \int_{0}^{t} \frac{w_{1} h}{w_{2}^{\prime} w_{1}-w_{2} w_{1}^{\prime}} d s \\
\quad+\left[\int_{t}^{1} \frac{w_{2} h}{w_{2}^{\prime} w_{1}-w_{2} w_{1}^{\prime}} d s+\frac{m}{w_{1}(1)}\right]\left((\hat{a}-L) w_{1}(t)+\left(N \operatorname{sgn}\left(w^{\prime}(t)\right)+\sqrt{L}\right) w_{1}^{\prime}(t)\right) \\
\leq W_{2}(t) \int_{0}^{t} \frac{w_{1} h}{w_{2}^{\prime} w_{1}-w_{2} w_{1}^{\prime}} d s+W_{1}(t)\left[\int_{t}^{1} \frac{w_{2} h}{w_{2}^{\prime} w_{1}-w_{2} w_{1}^{\prime}} d s+\frac{m}{w_{1}(1)}\right],
\end{aligned}
$$

for $t \in[0,1]$. The sign properties of $w_{1}, w_{2}, W_{1}, W_{2}$ and the assumptions on $h$ and $m$ immediately yield (30).

We introduce now a linear monotone iterative scheme for approximating the solution of (1); namely, we define by recurrence two sequences $\left(\alpha_{n}\right)_{n}$, and $\left(\beta_{n}\right)_{n}$ as follows:

- let $\alpha_{0}$ be any constant, with $\alpha_{0} \leq 0$, and, for $n \in \mathbb{N}$, let $\alpha_{n+1}$ be the solution of

$$
\left\{\begin{array}{l}
\alpha_{n+1}^{\prime \prime}+\sqrt{L} \alpha_{n+1}^{\prime}-L \alpha_{n+1}=\hat{f}\left(\alpha_{n}, \alpha_{n}^{\prime}\right)+\sqrt{L} \alpha_{n}^{\prime}-L \alpha_{n} \quad \text { in }[0,1] \\
\alpha_{n+1}^{\prime}(0)=\alpha_{n+1}(1)=0
\end{array}\right.
$$

- let $\beta_{0}$ be any constant, with $\beta_{0} \geq \frac{b}{a}$, and, for $n \in \mathbb{N}$, let $\beta_{n+1}$ be the solution of

$$
\left\{\begin{array}{l}
\beta_{n+1}^{\prime \prime}+\sqrt{L} \beta_{n+1}^{\prime}-L \beta_{n+1}=\hat{f}\left(\beta_{n}, \beta_{n}^{\prime}\right)+\sqrt{L} \beta_{n}^{\prime}-L \beta_{n} \quad \text { in }[0,1] \\
\beta_{n+1}^{\prime}(0)=\beta_{n+1}(1)=0 .
\end{array}\right.
$$

Theorem 2.5 Let $a>0$ and $b>0$ be given. Then there exists $L_{0}>0$, given by (39), such that for any $L \geq L_{0}$ the sequences $\left(\alpha_{n}\right)_{n}$ and $\left(\beta_{n}\right)_{n}$ recursively defined in (41) and (42), respectively, converge in $C^{2}([0,1])$ to the unique solution $u$ of $(21)$ and hence of $(1)$. In addition, for each $n \in \mathbb{N}$ the following conditions hold: 
- $\alpha_{n}$ is a strict lower solution and $\beta_{n}$ is a strict upper solution of (21), and

- $\alpha_{n} \ll \alpha_{n+1} \ll u \ll \beta_{n+1} \ll \beta_{n}$ in $[0,1]$.

Proof Let us fix $L \geq L_{0}$, where $L_{0}$ is given by (39).

Step 1 . The sequence $\left(\alpha_{n}\right)_{n}$ is such that, for each $n \in \mathbb{N}, \alpha_{n} \ll \alpha_{n+1}$ in $[0,1]$ and $\alpha_{n}$ is a strict lower solution of (21).

The proof is done by induction. Define, for each $n \in \mathbb{N}, u_{n+1}=\alpha_{n+1}-\alpha_{n}$. The function $u_{1}$ satisfies

$$
\left\{\begin{array}{l}
u_{1}^{\prime \prime}+\sqrt{L} u_{1}^{\prime}-L u_{1}=\hat{f}\left(\alpha_{0}, \alpha_{0}^{\prime}\right)-\alpha_{0}^{\prime \prime} \quad \text { in }[0,1] \\
u_{1}^{\prime}(0)=0 \\
u_{1}(1) \geq 0
\end{array}\right.
$$

Notice that $\hat{f}\left(\alpha_{0}, \alpha_{0}^{\prime}\right)-\alpha_{0}^{\prime \prime}<0$ in $[0,1]$. Hence the maximum principle implies that $u_{1} \gg 0$, that is, $\alpha_{0} \ll \alpha_{1}$, in $[0,1]$. Now, let us show that $\alpha_{1}$ is a strict lower solution of (21). Using the definition of $\alpha_{1}$, together with conditions $\left(\mathrm{h}_{1}\right)$ and $\left(\mathrm{h}_{2}\right)$, we get

$$
\begin{aligned}
\hat{f}\left(\alpha_{1}, \alpha_{1}^{\prime}\right)-\alpha_{1}^{\prime \prime} & =\left(\hat{f}\left(\alpha_{1}, \alpha_{1}^{\prime}\right)-\hat{f}\left(\alpha_{0}, \alpha_{0}^{\prime}\right)\right)+\sqrt{L} u_{1}^{\prime}-L u_{1} \\
& \leq\left(N\left|u_{1}^{\prime}\right|+\hat{a} u_{1}\right)+\sqrt{L} u_{1}^{\prime}-L u_{1} \\
& =(\hat{a}-L) u_{1}+\left(N \operatorname{sgn}\left(u_{1}^{\prime}\right)+\sqrt{L}\right) u_{1}^{\prime}
\end{aligned}
$$

in $[0,1]$. Since $u_{1}$ is a solution of (43), which is of the form of (28), with $h=\hat{f}\left(\alpha_{0}, \alpha_{0}^{\prime}\right)-\alpha_{0}^{\prime \prime}<0$ in $[0,1]$ and $m \geq 0$, Lemma 2.4 applies and yields

$$
(\hat{a}-L) u_{1}(t)+\left(N \operatorname{sgn}\left(u_{1}^{\prime}(t)\right)+\sqrt{L}\right) u_{1}^{\prime}(t)<0,
$$

for all $t \in[0,1]$. From (44), (45) and from the boundary conditions $\alpha_{1}^{\prime}(0)=\alpha_{1}(1)=0$, we conclude that $\alpha_{1}$ is a strict lower solution of (21).

Assume now that, for some integer $n \geq 1, \alpha_{n}$ is strict lower solution of (21) satisfying the boundary conditions. The function $u_{n+1}$ satisfies

$$
\left\{\begin{array}{l}
u_{n+1}^{\prime \prime}+\sqrt{L} u_{n+1}^{\prime}-L u_{n+1}=\hat{f}\left(\alpha_{n}, \alpha_{n}^{\prime}\right)-\alpha_{n}^{\prime \prime} \quad \text { in }[0,1] \\
u_{n+1}^{\prime}(0)=u_{n+1}(1)=0 .
\end{array}\right.
$$

As $\alpha_{n}$ is strict and satisfies the boundary conditions, we have $\hat{f}\left(\alpha_{n}, \alpha_{n}^{\prime}\right)-\alpha_{n}^{\prime \prime}<0$ in $[0,1]$. Hence the maximum principle yields $u_{n+1} \gg 0$, i.e., $\alpha_{n} \ll \alpha_{n+1}$ in $[0,1]$. Finally, $\alpha_{n+1}$ satisfies

$$
\begin{aligned}
\hat{f}\left(\alpha_{n+1}, \alpha_{n+1}^{\prime}\right)-\alpha_{n+1}^{\prime \prime} & =\left(\hat{f}\left(\alpha_{n+1}, \alpha_{n+1}^{\prime}\right)-\hat{f}\left(\alpha_{n}, \alpha_{n}^{\prime}\right)\right)+\sqrt{L} u_{n+1}^{\prime}-L u_{n+1} \\
& \leq\left(N\left|u_{n+1}^{\prime}\right|+\hat{a} u_{n+1}\right)+\sqrt{L} u_{n+1}^{\prime}-L u_{n+1} \\
& =(\hat{a}-L) u_{n+1}+\left(N \operatorname{sgn}\left(u_{n+1}^{\prime}\right)+\sqrt{L}\right) u_{n+1}^{\prime}
\end{aligned}
$$

in $[0,1]$. Since $u_{n+1}$ is the solution of problem (46), which is of the form of (28), with $h=\hat{f}\left(\alpha_{n}, \alpha_{n}^{\prime}\right)-\alpha_{n}^{\prime \prime}<0$ in $[0,1]$ and $m=0$, Lemma 2.4 applies and yields

$$
(\hat{a}-L) u_{n+1}(t)+\left(N \operatorname{sgn}\left(u_{n+1}^{\prime}(t)\right)+\sqrt{L}\right) u_{n+1}^{\prime}(t)<0,
$$


for all $t \in[0,1]$. From (47), (48), and from the boundary conditions $\alpha_{n+1}^{\prime}(0)=\alpha_{n+1}(1)=0$, we conclude that $\alpha_{n+1}$ is a strict lower solution of (21), such that $\hat{f}\left(\alpha_{n+1}, \alpha_{n+1}^{\prime}\right)-\alpha_{n+1}^{\prime \prime}<0$ in $[0,1]$.

In a similar way, one can prove the following conclusion.

Step 2. The sequence $\left(\beta_{n}\right)_{n}$ is such that, for each $n \in \mathbb{N}, \beta_{n+1} \ll \beta_{n}$ in $[0,1]$ and $\beta_{n}$ is a strict upper solution of (21).

Step 3. We have, for each $n \in \mathbb{N}, \alpha_{n} \ll \beta_{n}$ in $[0,1]$.

For each $n \in \mathbb{N}$, let us set

$$
z_{n}=\beta_{n}-\alpha_{n} \quad \text { and } \quad h_{n}=\hat{f}\left(\beta_{n}, \beta_{n}^{\prime}\right)-\hat{f}\left(\alpha_{n}, \alpha_{n}^{\prime}\right)+\sqrt{L} z_{n}^{\prime}-L z_{n}
$$

where, clearly, $z_{n+1}$ and $h_{n}$ satisfy

$$
\left\{\begin{array}{l}
z_{n+1}^{\prime \prime}+\sqrt{L} z_{n+1}^{\prime}-L z_{n+1}=h_{n} \quad \text { in }[0,1] \\
z_{n+1}^{\prime}(0)=z_{n+1}(1)=0 .
\end{array}\right.
$$

By construction, we have $z_{0} \gg 0$ in $[0,1]$ and

$$
h_{0}=\hat{f}\left(\beta_{0}, \beta_{0}^{\prime}\right)-\hat{f}\left(\alpha_{0}, \alpha_{0}^{\prime}\right)+\sqrt{L} z_{0}^{\prime}-L z_{0}=b\left(1-\frac{L}{a}\right) .
$$

As $L \geq L_{0}>\hat{a}>a$, we conclude that $h_{0}(t)<0$ for all $t \in[0,1]$.

Take now any $n \in \mathbb{N}$ and suppose that $z_{n} \gg 0$ and $h_{n}<0$ in $[0,1]$. From (49) we infer, using the maximum principle, that $z_{n+1} \gg 0$ in $[0,1]$. Let us prove that $h_{n+1}<0$ in $[0,1]$. We easily see that

$$
h_{n+1} \leq(\hat{a}-L) z_{n+1}+\left(N \operatorname{sgn}\left(z_{n+1}^{\prime}\right)+\sqrt{L}\right) z_{n+1}^{\prime}
$$

in $[0,1]$. As $z_{n+1}$ is the solution of problem (49), which is of the form of (28), with $h=h_{n}<0$ in $[0,1]$ and $m=0$, Lemma 2.4 applies and yields

$$
(\hat{a}-L) z_{n+1}(t)+\left(N \operatorname{sgn}\left(z_{n+1}^{\prime}(t)\right)+\sqrt{L}\right) z_{n+1}^{\prime}(t)<0
$$

and hence $h_{n+1}(t)<0$ for all $t \in[0,1]$. The conclusion $z_{n} \gg 0$, i.e., $\alpha_{n} \ll \beta_{n}$, in $[0,1]$ for all $n \in \mathbb{N}$, then follows by induction.

Step 4. There exists $C>0$ such that, for all $n \in \mathbb{N}$,

$$
\left\|\alpha_{n}^{\prime}\right\|_{\infty} \leq C \text { and }\left\|\beta_{n}^{\prime}\right\|_{\infty} \leq C
$$

We know that $\alpha_{1} \leq \alpha_{n} \leq \beta_{n} \leq \beta_{1}$ in $[0,1]$ for all $n \geq 1$, with $\alpha_{1}(1)=0=\beta_{1}(1)$. Hence we get

$$
-\alpha_{1}^{\prime}(1) \leq-\alpha_{n}^{\prime}(1) \leq-\beta_{n}^{\prime}(1) \leq-\beta_{1}^{\prime}(1)
$$

for all $n \geq 1$. Let us set $B=\left|\beta_{1}^{\prime}(1)\right|$.

Suppose, by contradiction, that (51) does not hold, i.e., for every $C>B$ there exists $j=$ $j(C) \in \mathbb{N}$ such that $\left\|\alpha_{j}^{\prime}\right\|_{\infty}>C$ or $\left\|\beta_{j}^{\prime}\right\|_{\infty}>C$. Assume that the former eventuality occurs. 
By Step 1 , using conditions $\left(\mathrm{h}_{1}\right)$ and $\left(\mathrm{h}_{2}\right)$, we get

$$
\alpha_{j}^{\prime \prime} \geq \hat{f}\left(\alpha_{j}, \alpha_{j}^{\prime}\right) \geq-N\left|\alpha_{j}^{\prime}\right|-b
$$

in $[0,1]$. Suppose that $\max _{[0,1]} \alpha_{j}^{\prime}>C$. Since $\alpha_{j}^{\prime}(0)=0$, there exists $\left.t_{C} \in\right] 0,1[$ such that $\alpha_{j}^{\prime}\left(t_{C}\right)=C$. Let $\left.\left.t_{B} \in\right] t_{C}, 1\right]$ be such that $\alpha_{j}^{\prime}\left(t_{B}\right)=B$ and $\left.\alpha_{j}^{\prime}(t) \in\right] B, C[$ for all $t \in] t_{C}, t_{B}[$. From (53) we infer

$$
1 \geq-\int_{t_{C}}^{t_{B}} \frac{\alpha_{j}^{\prime \prime}}{N\left|\alpha_{j}^{\prime}\right|+b} d t=\int_{B}^{C} \frac{d \xi}{N \xi+b}=\frac{1}{N}[\ln (N C+b)-\ln (N B+b)]
$$

The right-hand side of (54) diverges as $C \rightarrow+\infty$, then a contradiction follows.

In a completely similar way, we achieve the conclusion if $\min _{[0,1]} \alpha_{j}^{\prime}<-C$, or if $\left\|\beta_{j}^{\prime}\right\|_{\infty}>C$. Step 5. The sequence $\left(\alpha_{n}\right)_{n}$ converges in $C^{2}([0,1])$ to the solution $u$ of $(1)$.

It follows from the previous steps that the sequence $\left(\alpha_{n}\right)_{n}$ is increasing and bounded in $C^{2}([0,1])$. Therefore there exists a function $u:[0,1] \rightarrow \mathbb{R}$ which is the pointwise limit of $\left(\alpha_{n}\right)_{n}$ in $[0,1]$; in particular, $\alpha_{n} \ll u$ in $[0,1]$ for all $n \in \mathbb{N}$. Moreover, by the ArzelàAscoli theorem, any subsequence $\left(\alpha_{n_{l}}\right)_{l}$ of $\left(\alpha_{n}\right)_{n}$ admits a subsequence which is convergent in $C^{1}([0,1])$ to $u$. Then the whole sequence $\left(\alpha_{n}\right)_{n}$ converges in $C^{1}([0,1])$ to $u$. From the equation in (41) we see that the convergence takes place in $C^{2}([0,1])$. Hence $u$ is a solution of problem (21) and, by Lemma 2.3 and Theorem 2.2, it is in fact the unique solution of problem (1).

In a similar way, one can prove the following conclusion.

Step 6 . The sequence $\left(\beta_{n}\right)_{n}$ converges in $C^{2}([0,1])$ to the solution $u$ of $(1)$.

Thus the proof is completed.

Corollary 2.6 Let $a>0$ and $b>0$ be given. Then the unique solution $u$ of (21) is (Lyapunov) globally asymptotically stable as an equilibrium of the parabolic problem

$$
\begin{cases}\partial_{\tau} v-\partial_{t t} v+\hat{f}\left(v, v_{t}\right)=0 & \text { in }] 0,1[\times \mathbb{R} \\ \partial_{t} v(0, \tau)=v(1, \tau)=0 & \text { in } \mathbb{R}\end{cases}
$$

Proof Let us note that any lower, respectively upper, solution of (21) is a lower, respectively upper, solution of the parabolic problem

$$
\begin{cases}\partial_{\tau} v-\partial_{t t} v+\hat{f}\left(\nu, v_{t}\right)=0 & \text { in }] 0,1[\times \mathbb{R} \\ \partial_{t} v(0, \tau)=v(1, \tau)=0 & \text { in } \mathbb{R}, \\ \nu(t, \tau)=v(t, \tau+1) & \text { in }] 0,1[\times \mathbb{R} .\end{cases}
$$

Arguing as in the proof of Theorem 2.2 we see that $u$ is the unique solution of (56). Then Theorem 2.5 implies that $u$ is strictly order stable from below and from above. Actually, since any constant $\alpha_{0} \leq 0$ is a strict lower solution and any constant $\beta_{0} \geq \frac{b}{a}$ is a strict upper solution of (56), the results in [11, Section 2.6] imply that $u$ is (Lyapunov) globally asymptotically stable as a solution of (56) and hence as an equilibrium of (55). 
Table 1 Values of the approximations $\alpha_{n_{k}}, \beta_{n_{k}}$, defined by (41), (42) with $L=L_{0}$, such that $\left\|\beta_{n_{k}}-\alpha_{n_{k}}\right\|_{\infty}<10^{-k}$ for $k \in\{1,2,3\}$

\begin{tabular}{lllllll}
\hline & $\boldsymbol{t}=\mathbf{0}$ & $\boldsymbol{t}=\mathbf{0 . 2}$ & $\boldsymbol{t}=\mathbf{0 . 4}$ & $\boldsymbol{t}=\mathbf{0 . 6}$ & $\boldsymbol{t}=\mathbf{0 . 8}$ & $\boldsymbol{t}=\mathbf{1}$ \\
\hline $\boldsymbol{\beta}_{n_{1}}(t)$ & 0.436 & 0.421 & 0.373 & 0.290 & 0.167 & 0 \\
$\boldsymbol{\beta}_{n_{2}}(t)$ & 0.377 & 0.364 & 0.323 & 0.253 & 0.146 & 0 \\
$\boldsymbol{\beta}_{n_{3}}(t)$ & 0.371 & 0.358 & 0.319 & 0.249 & 0.144 & 0 \\
$\alpha_{n_{3}}(t)$ & 0.370 & 0.357 & 0.318 & 0.249 & 0.144 & 0 \\
$\alpha_{n_{2}}(t)$ & 0.367 & 0.354 & 0.315 & 0.247 & 0.143 & 0 \\
$\boldsymbol{\alpha}_{n_{1}}(t)$ & 0.336 & 0.325 & 0.289 & 0.227 & 0.132 & 0 \\
\hline
\end{tabular}

Remark 2.4 The definition of $\hat{f}$ implies that the solution $u$ of (1) is strictly order stable from below and from above and (Lyapunov) asymptotically stable as an equilibrium of the parabolic problem (11).

\section{Numerical experiments}

We present here some experiments concerning the numerical approximation of the solution of problem (1), for the same choice $a=b=1$ of the parameters as in [1].

The iterative scheme in case $L \geq L_{0} \quad$ We have computed various approximations, at different precision levels, of the unique solution $u$ of problem (1) by implementing in MatLab the linear iterative scheme defined by (41) and (42); at each step of the iteration the resulting linear equations have been solved using the bvp $4 \mathrm{c}$ routine with a 100-point grid. We have chosen $L=L_{0}$, with $L_{0}=1,828.5$ given by (39), $\alpha_{0}=0$ and $\beta_{0}=\frac{b}{a}=1$. Theorem 2.5 guarantees that the approximating sequences $\left(\alpha_{n}\right)_{n}$ and $\left(\beta_{n}\right)_{n}$ are constituted by lower and upper solutions and monotonically converge to $u$, in an increasing or decreasing fashion, respectively; thus, for each $n$, the couple $\alpha_{n}, \beta_{n}$ brackets the solution $u$, thus providing lower and upper estimates. In what follows the $L^{\infty}$-norm of a given function is intended to have been computed as the $L^{\infty}$-norm of its discretization on the given grid. We have denoted by $n_{k}$ the minimum number of iterations needed in order that $\left\|\beta_{n_{k}}-\alpha_{n_{k}}\right\|_{\infty}<10^{-k}$ for $k \in\{1,2,3\}$; the corresponding values are $n_{1}=1,421, n_{2}=2,788$ and $n_{3}=4,155$. In Table 1 we have tabulated $\alpha_{n_{k}}, \beta_{n_{k}}$, for $k \in\{1,2,3\}$, at the mesh points $t \in\{0,0.2,0.4,0.6,0.8,1\}$; the graphs of $\alpha_{n_{k}}, \beta_{n_{k}}$ are displayed in Figure 1; whereas Figure 2 describes the rate of decay of $\left\|\beta_{n}-\alpha_{n}\right\|_{\infty}$, as well as of the errors $\left\|\alpha_{n}-u\right\|_{\infty}$ and $\left\|\beta_{n}-u\right\|_{\infty}$, plotted against the number $n$ of iterations. Here $u$ denotes a reference approximation of the solution of (1), calculated using the same scheme up to a precision of $10^{-5}$. Although the lower solutions $\alpha_{n}$ converge slightly faster than the upper solutions $\beta_{n}$, it is evident that the monotone iterative scheme defined by (41) and (42) turns out to be extremely slow.

The iterative scheme in case $L \in\left[0, L_{0}[\right.$ We start from the obvious observation that the iterative scheme given by (41) and (42) is well defined for any fixed $L \geq 0$; hence it is clear that, if the resulting sequences $\left(\alpha_{n}\right)_{n}$ and $\left(\beta_{n}\right)_{n}$ are Cauchy sequences in $C^{1}([0,1])$, then, by the uniqueness of the solution of (1), they converge in $C^{2}([0,1])$ to $u$. Of course, if $L<L_{0}$ we cannot anymore guarantee that either $\alpha_{n}$ is a lower solution, or $\beta_{n}$ is an upper solution, or the sequences $\left(\alpha_{n}\right)_{n}$ and $\left(\beta_{n}\right)_{n}$ enjoy any monotonicity property. Let us take $\alpha_{0}=0$ in (41) and let $\left(\alpha_{n}\right)_{n}$ be the sequence of iterates obtained for some given $L \geq 0$. The numerical experiments, we have performed for several different choices of $L \in\left[0, L_{0}[\right.$, show 


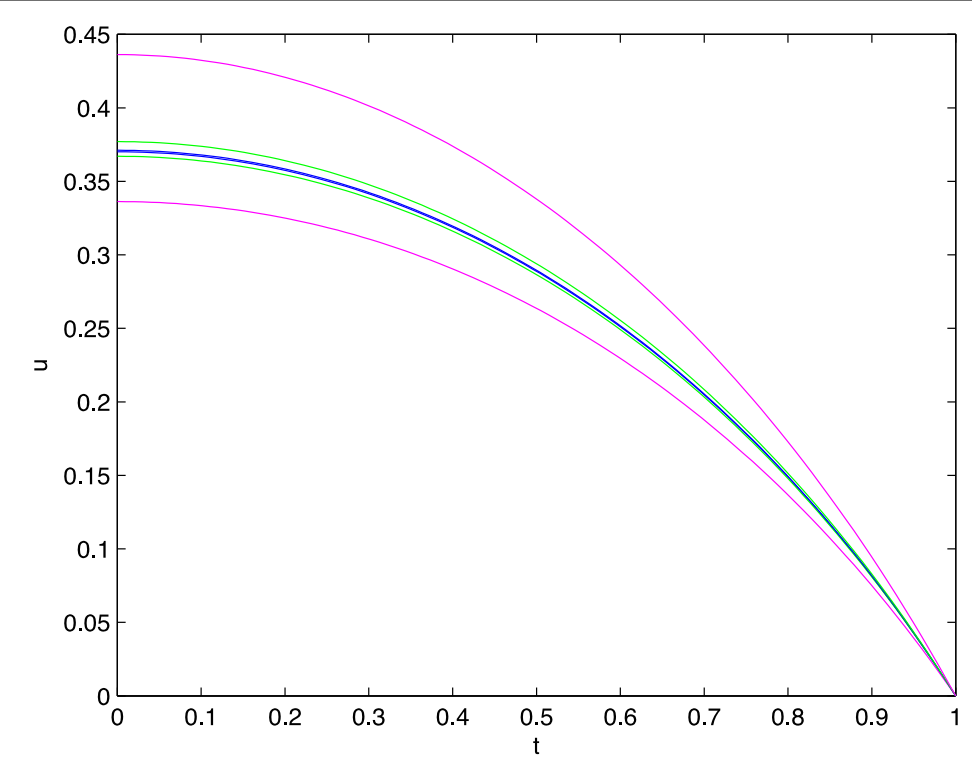

Figure 1 Graphs of the approximations $\alpha_{n_{1}}, \beta_{n_{1}}$ (in violet), $\alpha_{n_{2}}, \beta_{n_{2}}$ (in green) and $\alpha_{n_{3}}, \beta_{n_{3}}$ (in blue), with $\alpha_{n_{k}}, \beta_{n_{k}}$, defined by (41), (42) with $L=L_{0}$, such that $\left\|\beta_{n_{k}}-\alpha_{n_{k}}\right\|_{\infty}<10^{-k}$ for $k \in\{1,2,3\}$.

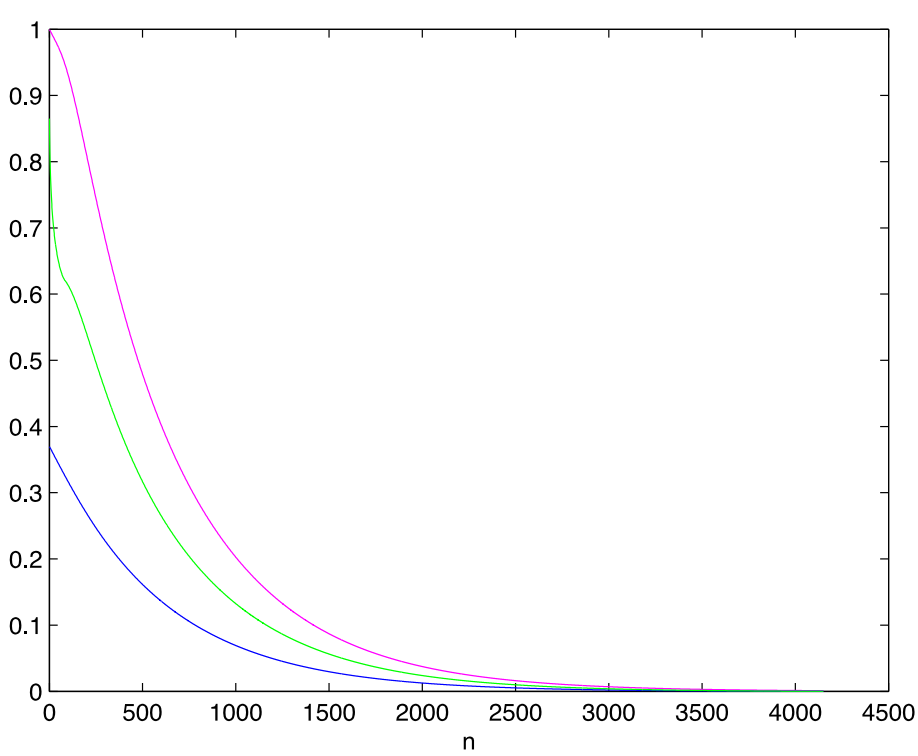

Figure 2 Graphs of $\left\|\alpha_{n}-u\right\|_{\infty}$ (in blue), $\left\|\beta_{n}-u\right\|_{\infty}$ (in green) and $\left\|\beta_{n}-\alpha_{n}\right\|_{\infty}$ (in violet), for $L=L_{0}$, plotted against the number $n$ of iterations.

that the sequence $\left(\alpha_{n}\right)_{n}$ converges to $u$, but the magnitude of $L$ strongly affects the speed of convergence; namely, as $L$ decreases, the required number of iterations $n$ in order that $\left\|\alpha_{n}-u\right\|_{\infty}$ goes beneath a prescribed threshold, decreases. In particular, the speed of convergence significantly increases as $L$ approximates 1 and, for this choice of $L$, it becomes comparable even with the speed of Newton's method. Indeed, if we fix an error tolerance of $10^{-3}$, the iterative scheme defined by (41), with $L=1$ and $\alpha_{0}=0$, converges in 4 iterations, whereas Newton's method, starting from $\alpha_{0}=0$ too, converges in 2 iterations: these re- 
Table 2 Values of the approximations $\alpha_{n}$, defined by (41) with $L=1$, for $n \in\{1,2,3,4\}$

\begin{tabular}{lllllll}
\hline & $\boldsymbol{t}=\mathbf{0}$ & $\boldsymbol{t}=\mathbf{0 . 2}$ & $\boldsymbol{t}=\mathbf{0 . 4}$ & $\boldsymbol{t}=\mathbf{0 . 6}$ & $\boldsymbol{t}=\mathbf{0 . 8}$ & $\boldsymbol{t}=\mathbf{1}$ \\
\hline$\alpha_{1}(t)$ & 0.284 & 0.271 & 0.233 & 0.175 & 0.097 & 0 \\
$\alpha_{2}(t)$ & 0.355 & 0.342 & 0.303 & 0.234 & 0.135 & 0 \\
$\alpha_{3}(t)$ & 0.370 & 0.357 & 0.318 & 0.250 & 0.149 & 0 \\
$\alpha_{4}(t)$ & 0.371 & 0.358 & 0.319 & 0.249 & 0.144 & 0 \\
\hline
\end{tabular}

Table 3 Values of the Newton approximations $u_{n}$ for $n \in\{1,2\}$

\begin{tabular}{lllllll}
\hline & $\boldsymbol{t}=\mathbf{0}$ & $\boldsymbol{t}=\mathbf{0 . 2}$ & $\boldsymbol{t}=\mathbf{0 . 4}$ & $\boldsymbol{t}=\mathbf{0 . 6}$ & $\boldsymbol{t}=\mathbf{0 . 8}$ & $\boldsymbol{t}=\mathbf{1}$ \\
\hline$u_{1}(t)$ & 0.352 & 0.339 & 0.298 & 0.229 & 0.129 & 0 \\
$u_{2}(t)$ & 0.370 & 0.358 & 0.318 & 0.249 & 0.144 & 0 \\
\hline
\end{tabular}

Table 4 Values of $u$ and $\tilde{u}$

\begin{tabular}{lllllll}
\hline & $\boldsymbol{t}=\mathbf{0}$ & $\boldsymbol{t}=\mathbf{0 . 2}$ & $\boldsymbol{t}=\mathbf{0 . 4}$ & $\boldsymbol{t}=\mathbf{0 . 6}$ & $\boldsymbol{t}=\mathbf{0 . 8}$ & $\boldsymbol{t}=\mathbf{1}$ \\
\hline$u(t)$ & 0.370 & 0.358 & 0.318 & 0.249 & 0.144 & 0 \\
$\tilde{u}(t)$ & 0.341 & 0.327 & 0.288 & 0.221 & 0.125 & 0
\end{tabular}

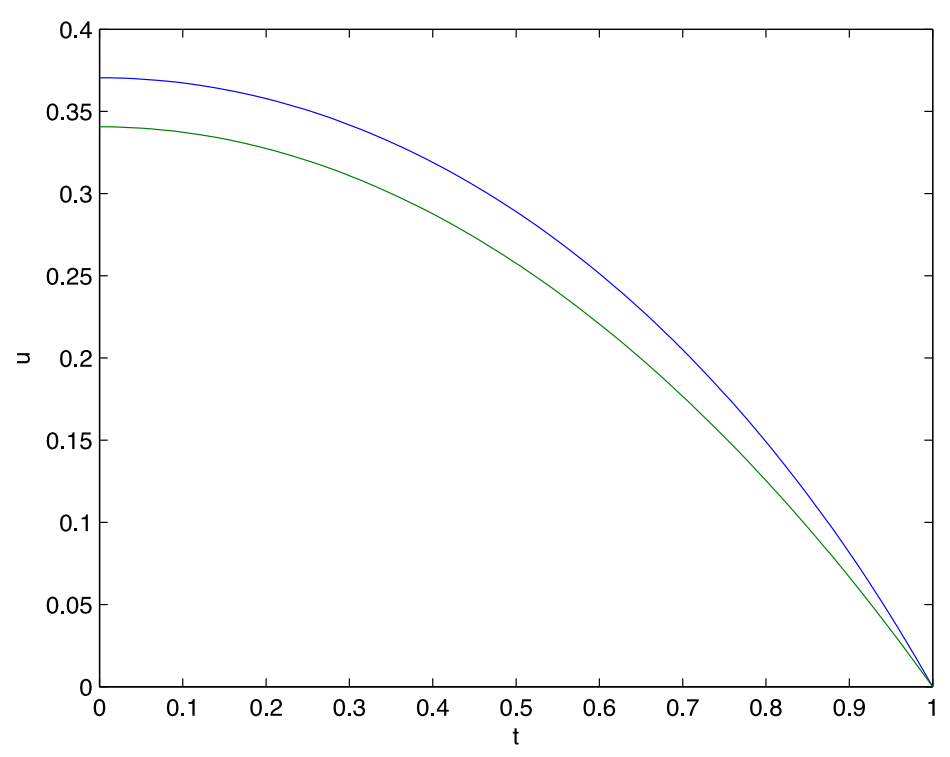

Figure 3 Graphs of $u$ (in blue) and $\tilde{u}$ (in green).

sults are displayed in Tables 2 and 3. This computational remark suggests the possibility of using the iterative scheme also in case the condition $L \geq L_{0}$ fails; however, its convergence properties should be theoretically analyzed.

A comparison between the solutions of (1) and (3) Here we present a numerical comparison between the solution $u$ of the fully nonlinear problem (1) and the solution $\tilde{u}$ of the partially linearized problem (3) investigated in [1]. We have approximated $u$ by the lower solution obtained by implementing the monotone iterative scheme given by (41), with $\alpha_{0}=0$, $L=L_{0}$ and stopping criterion $\left\|\beta_{n}-\alpha_{n}\right\|_{\infty}<10^{-5}$. An approximation of $\tilde{u}$, matching the one obtained in [1], has been calculated using the bvp $4 \mathrm{C}$ routine of MatLab with a 100-point 
grid. Table 4 reports the values of $u$ and $\tilde{u}$ at the mesh points $t \in\{0,0.2,0.4,0.6,0.8,1\}$ and Figure 3 displays the graphs of $u$ and $\tilde{u}$.

\section{Competing interests}

The authors declare that they have no competing interests.

\section{Authors' contributions}

All authors read and approved the final manuscript.

\section{Author details}

1 Area Departamental de Matemática, Instituto Superior de Engenharia de Lisboa, Rua Conselheiro Emídio Navarro 1, Lisboa, 1950-062, Portugal. ²Département de Mathématique, Université Libre de Bruxelles, CP 214 Boulevard du Triomphe, Bruxelles, 1052, Belgium. ${ }^{3}$ Dipartimento di Matematica e Geoscienze, Università degli Studi di Trieste, Via A. Valerio 12/1, Trieste, 34127, Italy

\section{Acknowledgements}

This paper was written under the auspices of INdAM-GNAMPA. The first named author has been supported by Fundação para a Ciência e a Tecnologia (SFRH/BD/61484/2009). The last two named authors have been supported by Università di Trieste, in the frame of the FRA projects 'Equazioni differenziali ordinarie: aspetti qualitativi e numerici' and 'Nonlinear Ordinary Differential Equations: Qualitative Theory, Numerics and Applications'. They also wish to thank Igor Moret for some useful discussions.

Received: 17 December 2013 Accepted: 2 May 2014 Published: 20 May 2014

\section{References}

1. Okrasiński, W, Płociniczak, Ł: A nonlinear mathematical model of the corneal shape. Nonlinear Anal., Real World Appl. 13, 1498-1505 (2012)

2. Okrasiński, W, Płociniczak, Ł: Bessel function model for corneal topography. Appl. Math. Comput. 223, 436-443 (2011)

3. Okrasiński, W, Płociniczak, Ł: Regularization of an ill-posed problem in corneal topography. Inverse Probl. Sci. Eng. 21, 1090-1097 (2013)

4. Płociniczak, Ł, Okrasiński, W, Nieto, JJ, Domínguez, O: On a nonlinear boundary value problem modeling corneal shape. J. Math. Anal. Appl. 414, 461-471 (2014)

5. De Coster, C, Habets, P: Two-Point Boundary Value Problems: Lower and Upper Solutions. Elsevier, Amsterdam (2006)

6. Le, VK: On a sub-supersolution method for the prescribed mean curvature problem. Czechoslov. Math. J. 58, 541-560 (2008)

7. Obersnel, F, Omari, P: Existence and multiplicity results for the prescribed mean curvature equation via lower and upper solutions. Differ. Integral Equ. 22, 853-880 (2009)

8. Obersnel, F, Omari, P, Rivetti, S: Existence, regularity and stability properties of periodic solutions of a capillarity equation in the presence of lower and upper solutions. Nonlinear Anal., Real World Appl. 13, 2830-2852 (2012)

9. Cherpion, M, De Coster, C, Habets, P: A constructive monotone iterative method for second-order BVP in the presence of lower and upper solutions. Appl. Math. Comput. 123, 75-91 (2001)

10. Cherpion, M: La Méthode des Sous et Sur-Solutions: Itérations Monotones et Problèmes Singuliers. Ph.D. thesis, Université Catholique de Louvain, Louvain-la-Neuve (2002)

11. De Coster, C, Obersnel, F, Omari, P: A qualitative analysis, via lower and upper solutions, of first order periodic evolutionary equations with lack of uniqueness. In: Canada, A, Drábek, P, Fonda, A (eds.) Handbook of Differential Equations: Ordinary Differential Equations, vol. III, pp. 203-339. Elsevier/North-Holland, Amsterdam (2006)

12. Ambrosetti, A, Malchiodi, A: Nonlinear Analysis and Semilinear Elliptic Problem. Cambridge University Press, New York (2007)

13. Hess, P: Periodic-Parabolic Boundary Value Problems and Positivity. Longman, Harlow (1991)

14. Daners, D, Koch Medina, P: Abstract Evolution Equations, Periodic Problems and Applications. Longman, Harlow (1992)

15. Kiguradze, IT: A priori estimates for the derivatives of bounded functions satisfying second-order differential inequalities. Differ. Uravn. 3, 1043-1052 (1967)

10.1186/1687-2770-2014-127

Cite this article as: Coelho et al.: A one-dimensional prescribed curvature equation modeling the corneal shape.

Boundary Value Problems 2014, 2014:127 